\title{
AUTOMORPHISMS OF THE QUOT SCHEMES ASSOCIATED TO COMPACT RIEMANN SURFACES
}

\author{
INDRANIL BISWAS, AJNEET DHILLON, AND JACQUES HURTUBISE
}

\begin{abstract}
Let $X$ be a compact connected Riemann surface of genus at least two. Fix positive integers $r$ and $d$. Let $\mathcal{Q}$ denote the Quot scheme that parametrizes the torsion quotients of $\mathcal{O}_{X}^{\oplus r}$ of degree $d$. This $\mathcal{Q}$ is also the moduli space of vortices for the standard action of $\mathrm{U}(r)$ on $\mathbb{C}^{r}$. The group $\operatorname{PGL}(r, \mathbb{C})$ acts on $\mathcal{Q}$ via the action of $\operatorname{GL}(r, \mathbb{C})$ on $\mathcal{O}_{X}^{\oplus r}$. We prove that this subgroup $\operatorname{PGL}(r, \mathbb{C})$ is the connected component, containing the identity element, of the holomorphic automorphism group $\operatorname{Aut}(\mathcal{Q})$. As an application of it, we prove that the isomorphism class of the complex manifold $\mathcal{Q}$ uniquely determines the isomorphism class of the Riemann surface $X$.
\end{abstract}

\section{INTRODUCTION}

Let $X$ be a compact connected Riemann surface of genus $g$, with $g \geq 2$. Fix positive integers $r$ and $d$. Let $\mathcal{Q}:=\operatorname{Quot}_{X}\left(\mathcal{O}_{X}^{\oplus r}, d\right)$ be the Quot scheme that parametrizes the torsion quotients

$$
\mathcal{O}_{X}^{\oplus r} \longrightarrow Q
$$

such that degree $(Q)=d$. It is a smooth complex projective manifold of dimension $r d$. Consider the standard action of $\mathrm{U}(r)$ on $\mathbb{C}^{r}$. The corresponding vortices are pairs of the form $(E, \phi)$, where $E$ is a holomorphic vector bundle on $X$ of rank $r$ and

$$
\phi: \mathcal{O}_{X}^{\oplus r} \longrightarrow E
$$

is a holomorphic homomorphism such that the subsheaf image $(\phi) \subset E$ is of rank $r$, equivalently, $E /$ image $(\phi)$ is a torsion sheaf [BDW]. Therefore, the dual homomorphism

$$
\phi^{*}: E^{*} \longrightarrow \mathcal{O}_{X}^{\oplus r}
$$

defines an element of $\mathcal{Q}$ if degree $(E)=d$. Consequently, $\mathcal{Q}$ is a moduli space of vortices on $X$.

Our aim here is to investigate the geometry of the variety $\mathcal{Q}$. Let $\operatorname{Aut}^{0}(\mathcal{Q})$ denote the connected component, containing the identity element, of the group of holomorphic automorphisms of $\mathcal{Q}$. The natural action of $\operatorname{GL}(r, \mathbb{C})$ on $\mathcal{O}_{X}^{\oplus r}$ produces an action of $\operatorname{PGL}(r, \mathbb{C})$ on $\mathcal{Q}$. let

$$
F: \operatorname{PGL}(r, \mathbb{C}) \longrightarrow \operatorname{Aut}^{0}(\mathcal{Q})
$$

be the homomorphism given by this action.

We prove the following (see Theorem 3.1 and Corollary [3.2):

2000 Mathematics Subject Classification. 14H60, 14J50.

Key words and phrases. Quot scheme, vortex moduli, vector field, automorphism, Torelli. 
Theorem 1.1. The homomorphism $F$ is an isomorphism. In particular, the homomorphism of Lie algebras

$$
s l(r, \mathbb{C}) \longrightarrow H^{0}(\mathcal{Q}, T \mathcal{Q})
$$

given by $F$ is an isomorphism.

Theorem 3.1 allows us to investigate the fixed point locus in $\mathcal{Q}$ for the action of a maximal torus of $\operatorname{Aut}^{0}(\mathcal{Q})$. As a consequence, we obtain the following Torelli type theorem (see Theorem 5.1):

Theorem 1.2. If $g=2=d$, assume that $r \geq 2$. Then the isomorphism class of the complex manifold $\mathcal{Q}$ uniquely determines the isomorphism class of the Riemann surface $X$.

The proof of Theorem 1.2 also uses a Torelli type theorem for $\operatorname{Sym}^{d}(X)$ proved in [Fa].

Take a holomorphic line bundle $L$ on $X$. Let $\mathcal{Q}\left(L^{\oplus r}, d\right)$ be the Quot scheme parametrizing torsion quotients of $L^{\oplus r}$ of dimension $d$. Then the variety $\mathcal{Q}\left(L^{\oplus r}, d\right)$ is canonically isomorphic to $\mathcal{Q}$. Therefore, Theorem 1.1 and Theorem 1.2 remain valid for $\mathcal{Q}\left(L^{\oplus r}, d\right)$.

\section{SElF-PRoduct, MEROMORPHIC FUnCTIONS AND MEROMORPHIC VECTOR FIELDS}

Let $X$ be a compact connected Riemann surface of genus $g$, with $g \geq 2$. The Cartesian product $X \times X$ will be denoted by $X^{2}$. Let $\Delta \subset X^{2}$ be the diagonal consisting of points of the form $(x, x)$ with $x \in X$. For $\ell=1,2$, let

$$
p_{\ell}: X^{2} \longrightarrow X
$$

be the projection to the $\ell$-th factor. The holomorphic tangent bundle of $X$ will be denoted by $T X$.

Lemma 2.1. For any $i \geq 0$,

$$
H^{0}\left(X^{2}, \mathcal{O}_{X^{2}}(i \cdot \Delta)\right)=H^{0}\left(X^{2}, \mathcal{O}_{X^{2}}\right)=\mathbb{C} .
$$

For any $i \geq 0$,

$$
H^{0}\left(X^{2},\left(p_{1}^{*} T X\right)^{\otimes \alpha_{1}} \otimes\left(p_{2}^{*} T X\right)^{\otimes \alpha_{2}} \otimes \mathcal{O}_{X^{2}}(i \cdot \Delta)\right)=0,
$$

where $\alpha_{1}$ and $\alpha_{2}$ are nonnegative integers with $\alpha_{1}+\alpha_{2}>0$.

Proof. Let $\iota: X \longrightarrow X^{2}$ be the map defined by $x \longmapsto(x, x)$. It identifies $X$ with $\Delta$. From the Poincaré adjunction formula we know that $\iota^{*}\left(\left.\mathcal{O}_{X^{2}}(\Delta)\right|_{\Delta}\right)=T X$; see [GH, p. 146] for Poincaré adjunction formula.

For any $i \geq 0$, consider the short exact sequence of coherent analytic sheaves on $X^{2}$

$\left.0 \longrightarrow \mathcal{O}_{X^{2}}(i \cdot \Delta) \longrightarrow \mathcal{O}_{X^{2}}((i+1) \cdot \Delta) \longrightarrow \mathcal{O}_{X^{2}}((i+1) \cdot \Delta)\right|_{\Delta}=\iota_{*}\left((T X)^{\otimes(i+1)}\right) \longrightarrow 0$.

Let

$$
H^{0}\left(X^{2}, \mathcal{O}_{X^{2}}(i \cdot \Delta)\right) \longrightarrow H^{0}\left(X^{2}, \mathcal{O}_{X^{2}}((i+1) \cdot \Delta)\right) \longrightarrow H^{0}\left(X,(T X)^{\otimes(i+1)}\right)
$$


be the corresponding long exact sequence of cohomologies. We have

$$
H^{0}\left(X,(T X)^{\otimes(i+1)}\right)=0
$$

because $g \geq 2$. Hence the above homomorphism

$$
H^{0}\left(X^{2}, \mathcal{O}_{X^{2}}(i \cdot \Delta)\right) \longrightarrow H^{0}\left(X^{2}, \mathcal{O}_{X^{2}}((i+1) \cdot \Delta)\right)
$$

is an isomorphism. Now using downward induction on $i$,

$$
H^{0}\left(X^{2}, \mathcal{O}_{X^{2}}(i \cdot \Delta)\right)=H^{0}\left(X^{2}, \mathcal{O}_{X^{2}}\right)=\mathbb{C} .
$$

This proves the first part of the lemma.

To prove the second part of the lemma, consider the short exact sequence of coherent analytic sheaves on $X^{2}$

$$
\begin{aligned}
0 \longrightarrow\left(p_{1}^{*} T X\right)^{\otimes \alpha_{1}} \otimes\left(p_{2}^{*} T X\right)^{\otimes \alpha_{2}} & \otimes \mathcal{O}_{X^{2}}(i \cdot \Delta) \longrightarrow\left(p_{1}^{*} T X\right)^{\otimes \alpha_{1}} \otimes\left(p_{2}^{*} T X\right)^{\otimes \alpha_{2}} \otimes \mathcal{O}_{X^{2}}((i+1) \cdot \Delta) \\
& \longrightarrow \iota_{*}\left((T X)^{\otimes\left(\alpha_{1}+\alpha_{2}+i+1\right)}\right) \longrightarrow 0
\end{aligned}
$$

obtained by tensoring (11) with $\left(p_{1}^{*} T X\right)^{\otimes \alpha_{1}} \otimes\left(p_{2}^{*} T X\right)^{\otimes \alpha_{2}}$. Since $H^{0}\left(X,(T X)^{\otimes m}\right)=0$ for all $m \geq 1$, using downward induction on $i$, we have $H^{0}\left(X^{2},\left(p_{1}^{*} T X\right)^{\otimes \alpha_{1}} \otimes\left(p_{2}^{*} T X\right)^{\otimes \alpha_{2}} \otimes \mathcal{O}_{X^{2}}(i \cdot \Delta)\right)=H^{0}\left(X^{2},\left(p_{1}^{*} T X\right)^{\otimes \alpha_{1}} \otimes\left(p_{2}^{*} T X\right)^{\otimes \alpha_{2}}\right)=0$.

This completes the proof.

Take any integer $n \geq 1$. Let

$$
X^{n}:=\overbrace{X \times \cdots \times X}^{n \text {-times }}
$$

be the $n$-fold Cartesian product. The projection of $X^{n}$ to the $\ell$-th factor, $1 \leq \ell \leq n$, will be denoted by $p_{\ell}$. For $1 \leq j<k \leq n$, let

$$
\Delta_{j, k} \subset X^{n}
$$

be the divisor consisting of points whose of $X^{n} j$-th coordinate coincides with the $k$-th coordinate.

Take integers $m_{j, k} \geq 0$, where $1 \leq j<k \leq n$. Fix a pair $\left(j_{0}, k_{0}\right)$, with $1 \leq j_{0}<$ $k_{0} \leq n$. Define $m_{j, k}^{\prime}, 1 \leq j<k \leq n$, as follows:

- $m_{j_{0}, k_{0}}^{\prime}=m_{j_{0}, k_{0}}+1$, and

$$
\text { - } m_{j, k}^{\prime}=m_{j, k} \text { if }(j, k) \neq\left(j_{0}, k_{0}\right) \text {. }
$$

Lemma 2.2. For any $i \geq 0$, the natural inclusion

$$
H^{0}\left(X^{n}, \mathcal{O}_{X^{n}}\left(\sum_{1 \leq j<k \leq n} m_{j, k} \cdot \Delta_{j, k}\right)\right) \hookrightarrow H^{0}\left(X^{n}, \mathcal{O}_{X^{n}}\left(\sum_{1 \leq j<k \leq n} m_{j, k}^{\prime} \cdot \Delta_{j, k}\right)\right)
$$

is an isomorphism.

For nonnegative integers $\alpha_{\ell}, \ell \in\{1, \cdots, n\}$, with $\sum_{\ell=1}^{n} \alpha_{\ell}>0$, the natural inclusion

$$
H^{0}\left(X^{n},\left(\bigotimes_{\ell=1}^{n}\left(p_{\ell}^{*} T X\right)^{\otimes \alpha_{\ell}}\right) \otimes \mathcal{O}_{X^{n}}\left(\sum_{1 \leq j<k \leq n} m_{j, k} \cdot \Delta_{j, k}\right)\right)
$$


is an isomorphism.

$$
\hookrightarrow H^{0}\left(X^{n},\left(\bigotimes_{\ell=1}^{n}\left(p_{\ell}^{*} T X\right)^{\otimes \alpha_{\ell}}\right) \otimes \mathcal{O}_{X^{n}}\left(\sum_{1 \leq j<k \leq n} m_{j, k}^{\prime} \cdot \Delta_{j, k}\right)\right)
$$

Proof. If $n=1$, then $\Delta_{j, k}$ are the zero divisors. Hence the lemma holds for $n=1$ as $g \geq 2$. If $n=2$, then it reduces to Lemma 2.1. We will prove the lemma using induction on $n$.

Assume that the lemma is proved for all $n \leq a-1$. For any $\ell \in\{1, \cdots, a-1\}$, let

$$
q_{\ell}: X^{a-1} \longrightarrow X
$$

be the projection to the $\ell$-th factor. Fix nonnegative integers $n_{j, k}$ for every $1 \leq j<k \leq$ $a-1$. Since the first statement of the lemma holds for $n=a-1$, we conclude that

$$
H^{0}\left(X^{a-1}, \mathcal{O}_{X^{a-1}}\left(\sum_{1 \leq j<k \leq a-1} n_{j, k} \cdot \Delta_{j, k}\right)\right)=\mathbb{C},
$$

where $\Delta_{j, k} \subset X^{a-1}$ is the divisor defined in (2). Since the second statement of the lemma holds for $n=a-1$, we conclude that

$$
H^{0}\left(X^{a-1},\left(\bigotimes_{\ell=1}^{a-1}\left(q_{\ell}^{*} T X\right)^{\otimes \alpha_{\ell}}\right) \otimes \mathcal{O}_{X^{a-1}}\left(\sum_{1 \leq j<k \leq a-1} n_{j, k} \cdot \Delta_{j, k}\right)\right)=0,
$$

where $\alpha_{\ell}$ are nonnegative integers with $\sum_{\ell=1}^{a-1} \alpha_{\ell}>0$.

We will prove the lemma for $n=a$.

Take $\left(j_{0}, k_{0}\right),\left\{m_{j, k}\right\}$ and $\left\{m_{j, k}^{\prime}\right\}$ as in the lemma. Let

$$
\iota: X^{a-1} \longrightarrow X^{a}=X^{n}
$$

be the map that sends $\left(x_{1}, \cdots, x_{a-1}\right)$ to $\left(y_{1}, \cdots, y_{a}\right)$ such that

- $y_{c}=x_{c}$ if $c \leq k_{0}-1$,

- $y_{k_{0}}=x_{j_{0}}$, and

- $y_{c}=x_{c-1}$ if $c>k_{0}$.

So $\iota$ identifies $X^{a-1}$ with $\Delta_{j_{0}, k_{0}}$. The Poincaré adjunction formula says that

$$
\iota^{*}\left(\left.\mathcal{O}_{X^{a}}\left(\Delta_{j_{0}, k_{0}}\right)\right|_{\Delta_{j_{0}, k_{0}}}\right)=\left(q_{j_{0}}\right)^{*} T X,
$$

where $q_{j_{0}}$ is defined in (3). For any $(j, k) \neq\left(j_{0}, k_{0}\right)$, let

$$
\Delta_{j, k}^{\prime}:=\iota^{-1}\left(\Delta_{j, k} \cap \Delta_{j_{0}, k_{0}}\right) \subset X^{a-1}
$$

be the diagonal divisor.

Consider the short exact sequence of coherent analytic sheaves on $X^{a}$

$$
\begin{gathered}
\left.0 \longrightarrow \mathcal{O}_{X^{a}}\left(\sum_{1 \leq j<k \leq a} m_{j, k} \cdot \Delta_{j, k}\right) \longrightarrow \mathcal{O}_{X^{a}}\left(\sum_{1 \leq j<k \leq a} m_{j, k}^{\prime} \cdot \Delta_{j, k}\right) \longrightarrow\left(\mathcal{O}_{X^{a}}\left(\sum_{1 \leq j<k \leq a} m_{j, k}^{\prime} \cdot \Delta_{j, k}\right)\right)\right|_{\Delta_{j_{0}, k_{0}}} \\
=\iota_{*}\left(\left(q_{j_{0}}\right)^{*}(T X)^{\otimes m_{j_{0}, k_{0}}^{\prime}} \otimes \mathcal{O}_{X^{a-1}}\left(\sum_{(j, k) \neq\left(j_{0}, k_{0}\right)} m_{j, k}^{\prime} \cdot \Delta_{j, k}^{\prime}\right)\right) \longrightarrow 0
\end{gathered}
$$


where $\Delta_{j, k}^{\prime}$ is defined in (7) and $q_{j_{0}}$ is defined in (3); the identification

$$
\begin{gathered}
\left.\mathcal{O}_{X^{a}}\left(\sum_{1 \leq j<k \leq a} m_{j, k}^{\prime} \cdot \Delta_{j, k}\right) \longrightarrow\left(\mathcal{O}_{X^{a}}\left(\sum_{1 \leq j<k \leq a} m_{j, k}^{\prime} \cdot \Delta_{j, k}\right)\right)\right|_{\Delta_{j_{0}, k_{0}}} \\
=\iota_{*}\left(\left(q_{j_{0}}\right)^{*}(T X)^{\otimes m_{j_{0}, k_{0}}^{\prime}} \otimes \mathcal{O}_{X^{a-1}}\left(\sum_{(j, k) \neq\left(j_{0}, k_{0}\right)} m_{j, k}^{\prime} \cdot \Delta_{j, k}^{\prime}\right)\right)
\end{gathered}
$$

in (8) is constructed using the isomorphism in (6). From (5) we know that

$$
H^{0}\left(X^{a-1},\left(p_{j_{0}}^{\prime}\right)^{*}(T X)^{\otimes m_{j_{0}, k_{0}}^{\prime}} \otimes \mathcal{O}_{X^{a-1}}\left(\sum_{(j, k) \neq\left(j_{0}, k_{0}\right)} m_{j, k}^{\prime} \cdot \Delta_{j, k}^{\prime}\right)\right)=0 .
$$

Therefore, from the long exact sequence of cohomologies associated to the short exact sequence in (8) we conclude that

$$
H^{0}\left(X^{a}, \mathcal{O}_{X^{a}}\left(\sum_{1 \leq j<k \leq a} m_{j, k} \cdot \Delta_{j, k}\right)\right)=H^{0}\left(X^{a}, \mathcal{O}_{X^{a}}\left(\sum_{1 \leq j<k \leq a} m_{j, k}^{\prime} \cdot \Delta_{j, k}\right)\right) .
$$

This proves the first statement of the lemma for $n=a$. Therefore, the proof of the first statement of the lemma is complete by induction on $n$.

We will now prove the second statement. Take $\left\{\alpha_{\ell}\right\}$ as in the second statement of the lemma. For $\ell \in\{1, \cdots, a-1\}$, define $\alpha_{\ell}^{\prime}$ as follows:

- $\alpha_{\ell}^{\prime}=\alpha_{\ell}$ if $\ell<j_{0}$,

- $\alpha_{j_{0}}^{\prime}=\alpha_{j_{0}}+\alpha_{k_{0}}$,

- $\alpha_{\ell}^{\prime}=\alpha_{\ell}$ if $j_{0}<\ell<k_{0}$, and

- $\alpha_{\ell}^{\prime}=\alpha_{\ell+1}$ if $\ell \geq k_{0}$.

Note that $\sum_{\ell=1}^{a} \alpha_{\ell}=\sum_{\ell=1}^{a-1} \alpha_{\ell}^{\prime}$.

Let

$$
\begin{aligned}
0 & \longrightarrow\left(\bigotimes_{\ell=1}^{a}\left(p_{\ell}^{*} T X\right)^{\otimes \alpha_{\ell}}\right) \otimes \mathcal{O}_{X^{a}}\left(\sum_{1 \leq j<k \leq a} m_{j, k} \cdot \Delta_{j, k}\right) \longrightarrow\left(\bigotimes_{\ell=1}^{a}\left(p_{\ell}^{*} T X\right)^{\otimes \alpha_{\ell}}\right) \otimes \mathcal{O}_{X^{a}}\left(\sum_{1 \leq j<k \leq a} m_{j, k}^{\prime} \cdot \Delta_{j, k}\right) \\
& \longrightarrow \iota_{*}\left(\left(\bigotimes_{\ell=1}^{a-1}\left(q_{\ell}^{*} T X\right)^{\otimes \alpha_{\ell}^{\prime}}\right) \otimes\left(q_{j_{0}}\right)^{*}(T X)^{\otimes m_{j_{0}, k_{0}}^{\prime}} \otimes \mathcal{O}_{X^{a-1}}\left(\sum_{(j, k) \neq\left(j_{0}, k_{0}\right)} m_{j, k}^{\prime} \cdot \Delta_{j, k}^{\prime}\right)\right) \longrightarrow 0,
\end{aligned}
$$

be the short exact sequence of coherent analytic sheaves on $X^{a}$ obtained by tensoring (8) with $\bigotimes_{\ell=1}^{a}\left(p_{\ell}^{*} T X\right)^{\otimes \alpha_{\ell}}$. From (5) we know that

$$
H^{0}\left(X^{a-1},\left(\bigotimes_{\ell=1}^{a-1}\left(q_{\ell}^{*} T X\right)^{\otimes \alpha_{\ell}^{\prime}}\right) \otimes\left(q_{j_{0}}\right)^{*}(T X)^{\otimes m_{j_{0}, k_{0}}^{\prime}} \otimes \mathcal{O}_{X^{a-1}}\left(\sum_{(j, k) \neq\left(j_{0}, k_{0}\right)} m_{j, k}^{\prime} \cdot \Delta_{j, k}^{\prime}\right)\right)=0
$$

Therefore, from the long exact sequence of cohomologies associated to the above short exact sequence of sheaves we conclude that the second statement of the lemma holds for $n=a$. This completes the proof by induction on $n$.

Proposition 2.3. For any $n \geq 1$ and nonnegative integers $m_{j, k}, 1 \leq j<k \leq n$,

$$
H^{0}\left(X^{n}, T X^{n} \otimes \mathcal{O}_{X^{n}}\left(\sum_{1 \leq j<k \leq n} m_{j, k} \cdot \Delta_{j, k}\right)\right)=0
$$


where $T X^{n}$ is the holomorphic tangent bundle of $X^{n}$.

Proof. Since

$$
T X^{n}=\bigoplus_{\ell=1}^{n} p_{\ell}^{*} T X,
$$

where $p_{\ell}$ is the projection of $X^{n}$ to the $\ell$-th factor, the proposition follows from the second statement in Lemma 2.2,

\section{Vector FIELdS on the Quot SChEme}

Let $E^{0}:=\mathcal{O}_{X}^{\oplus r}$ be the trivial holomorphic vector bundle over $X$ of rank $r$. Fix a positive integer $d$. Let

$$
\mathcal{Q}:=\operatorname{Quot}_{X}\left(E^{0}, d\right)
$$

be the Quot scheme that parametrizes the torsion quotients of $E^{0}$ of dimension $d$. So each point of $\mathcal{Q}$ represents a quotient

$$
\varphi: E^{0} \rightarrow Q
$$

of the $\mathcal{O}_{X}$-module $E^{0}$ such that

- $Q$ is a torsion $\mathcal{O}_{X}$-module, and

- $\operatorname{dim} H^{0}(X, Q)=d$.

The obstruction to the smoothness of the variety $\mathcal{Q}$ at the point $Q \in \mathcal{Q}$ is given by $\operatorname{Ext}_{\mathcal{O}_{X}}^{1}(\operatorname{kernel}(\varphi), Q)[\mathrm{Be}$, p. 1, Theorem 2]. For any $\varphi$ as in (9), since $\operatorname{kernel}(\varphi)$ is locally free, and the dimension of the support of $Q$ is zero. we have

$$
\operatorname{Ext}_{\mathcal{O}_{X}}^{1}(\operatorname{kernel}(\varphi), Q)=0 \text {. }
$$

Therefore, $\mathcal{Q}$ is an irreducible smooth complex projective variety. Its dimension is $r d$.

Consider the tautological action of the group $\operatorname{Aut}\left(E^{0}\right)=\mathrm{GL}(r, \mathbb{C})$ on $E^{0}$. It produces an effective action of $\operatorname{PGL}(r, \mathbb{C})$ on $\mathcal{Q}$

$$
\operatorname{PGL}(r, \mathbb{C}) \times \mathcal{Q} \longrightarrow \mathcal{Q} .
$$

Consider the Lie algebra $s l(r, \mathbb{C})$ (the space of $r \times r$ complex matrices of trace zero) of $\operatorname{PGL}(r, \mathbb{C})$. Let

$$
\gamma: \operatorname{sl}(r, \mathbb{C}) \longrightarrow H^{0}(\mathcal{Q}, T \mathcal{Q})
$$

be the homomorphism of Lie algebras given by the action of $\operatorname{PGL}(r, \mathbb{C})$ on $\mathcal{Q}$ in (10). (The Lie algebra structure of $H^{0}(\mathcal{Q}, T \mathcal{Q})$ is given by Lie bracket of vector fields.)

Theorem 3.1. The homomorphism $\gamma$ in (11) is an isomorphism.

Proof. The homomorphism $\gamma$ is injective because the homomorphism from $s l(r, \mathbb{C})$ to the space of holomorphic vector fields on $\mathbb{C P}^{r-1}$, given by the standard action of $\operatorname{PGL}(r, \mathbb{C})$ on $\mathbb{C P}^{r-1}$, is injective. 
Let $\operatorname{Sym}^{d}(X)$ be the $d$-fold symmetric product of $X$. It parametrizes the effective divisors on $X$ of degree $d$. Let

$$
U \subset \operatorname{Sym}^{d}(X)
$$

be the Zariski open subset parametrizing distinct $d$ points; so $U$ parametrizes the reduced effective divisors of degree $d$. The group of permutations of $\{1, \cdots, d\}$ will be denoted by $S_{d}$. Let

$$
f: \widetilde{U}:=X^{d} \backslash\left(\bigcup_{1 \leq j<k \leq d} \Delta_{j, k}\right) \longrightarrow U
$$

be natural projection, where $\Delta_{j, k}$ is defined in (2). We note that $f$ is an étale Galois covering with Galois group $S_{d}$.

Sending any quotient $Q \in \mathcal{Q}$ of $E^{0}$ to the scheme-theoretic support of $Q$, we obtain a morphism

$$
\widetilde{\beta}: \mathcal{Q} \longrightarrow \operatorname{Sym}^{d}(X) \text {. }
$$

Define

$$
\mathcal{U}:=\widetilde{\beta}^{-1}(U) \subset \mathcal{Q} .
$$

The restriction of $\widetilde{\beta}$ to $\mathcal{U}$ will be denoted by $\beta$. We note that

$$
\beta: \mathcal{U} \longrightarrow U
$$

is a smooth proper surjective morphism. The fiber of $\beta$ over any $z \in U$ is the Cartesian product

$$
\mathcal{U}_{z}:=\beta^{-1}(z)=\prod_{x \in z} P\left(E_{x}^{0}\right) .
$$

So $\mathcal{U}_{z}$ is isomorphic to $\left(\mathbb{C P}^{r-1}\right)^{d}$.

Take any

$$
\theta \in H^{0}(\mathcal{Q}, T \mathcal{Q}) .
$$

Let $\theta_{0}$ be the restriction of $\theta$ to $\mathcal{U}$. Let

$$
d \beta: T \mathcal{U} \longrightarrow \beta^{*} T U
$$

be the differential of the projection $\beta$ in (14). Since the fibers of $\beta$ are connected and projective, we conclude that $d \beta\left(\theta_{0}\right)$ is the pullback of a holomorphic vector field on $U$. Let $\theta^{\prime}$ be the holomorphic vector field on $U$ such that

$$
d \beta\left(\theta_{0}\right)=\beta^{*} \theta^{\prime} .
$$

Let

$$
\theta^{\prime \prime}:=f^{*} \theta^{\prime} \in H^{0}(\widetilde{U}, T \widetilde{U})
$$

be the pullback, where $f$ is the projection in (12). Since $\theta_{0}$ is the restriction of a holomorphic vector field on $\mathcal{Q}$, it follows that $\theta^{\prime \prime}$ is meromorphic on $X^{d}$, or in other words,

$$
\theta^{\prime \prime} \in H^{0}\left(X^{d}, T X^{d} \otimes \mathcal{O}_{X^{d}}\left(\sum_{1 \leq j<k \leq d} m_{j, k} \cdot \Delta_{j, k}\right)\right)
$$


for sufficiently large integers $m_{j, k}$. Therefore, from Proposition 2.3 we conclude that $\theta^{\prime \prime}=0$. Hence

$$
d \beta\left(\theta_{0}\right)=0 .
$$

In other words, $\theta_{0}$ is vertical for the projection $\beta$.

Let

$$
\operatorname{ad}\left(E^{0}\right) \subset \operatorname{End}\left(E^{0}\right)=E^{0} \otimes\left(E^{0}\right)^{*}
$$

be the subbundle of rank $r^{2}-1$ defined by the sheaf of endomorphisms of trace zero. For any point $x \in X$, we have

$$
H^{0}\left(P\left(E_{x}^{0}\right), T P\left(E_{x}^{0}\right)\right)=\operatorname{ad}\left(E^{0}\right)_{x}=\operatorname{sl}(r, \mathbb{C}) .
$$

In view of (15),

$$
H^{0}\left(\mathcal{U}_{z}, T \mathcal{U}_{z}\right)=\bigoplus_{x \in z} \operatorname{ad}\left(E^{0}\right)_{x}=\bigoplus_{x \in z} \operatorname{sl}(r, \mathbb{C})
$$

for all $z \in U$. We will show that the restriction to $\mathcal{U}_{z}$ of any holomorphic vector field on $\mathcal{Q}$ is a constant diagonal element of $\bigoplus_{x \in z} \operatorname{sl}(r, \mathbb{C})$ which is independent of $z$.

Let

$$
\mathcal{Z}:=f^{*} \mathcal{Q}=\widetilde{U} \times_{U} \mathcal{U} \longrightarrow \widetilde{U}
$$

be the pullback to $\widetilde{U}$ of the fiber bundle $\beta: \mathcal{U} \longrightarrow U$. The natural projection

$$
\phi: \mathcal{Z}:=f^{*} \mathcal{Q} \longrightarrow \mathcal{U}
$$

is an étale Galois covering with Galois group $S_{d}$.

As before, let $\theta_{0}$ be the restriction to $\mathcal{U}$ of a holomorphic vector field on $\mathcal{Q}$. Consider the vector field

$$
\theta_{1}:=\phi^{*} \theta_{0} \in H^{0}(\mathcal{Z}, T \mathcal{Z})
$$

on $\mathcal{Z}$, where $\phi$ is the projection in (16). We know that $\theta_{1}$ is vertical for the projection $\mathcal{Z} \longrightarrow \widetilde{U}$.

Note that the fibers of the projection $\mathcal{Z} \longrightarrow \widetilde{U}$ are canonically identified with $\left(\mathbb{C P}^{r-1}\right)^{d}$. Therefore, the vector field $\theta_{1}$ is a holomorphic function on $\widetilde{U}$ with values in $\operatorname{sl}(r, \mathbb{C})^{\oplus d}$. This holomorphic function is meromorphic on $X^{d}$ because $\theta_{0}$ is the restriction to $\mathcal{U}$ of a holomorphic vector field on $\mathcal{Q}$. From the first part of Lemma 2.2 we know that there are no nonconstant meromorphic functions on $X^{d}$ that are holomorphic on $\widetilde{U}$. Hence $\theta_{1}$ is a constant function from $\widetilde{U}$ to $\operatorname{sl}(r, \mathbb{C})^{\oplus d}$. This function $\widetilde{U} \longrightarrow \operatorname{sl}(r, \mathbb{C})^{\oplus d}$ has to be invariant under the action of the Galois group $S_{d}$ on $\widetilde{U}$ because $\theta_{1}$ is the pullback of a vector field on $\mathcal{U}$. Therefore, there is an element

$$
v \in \operatorname{sl}(r, \mathbb{C})
$$

such that $\theta_{1}$ is the constant function $(v, \cdots, v)$. Since $\mathcal{U}$ is dense in $\mathcal{Q}$, this immediately implies that the injective homomorphism $\gamma$ in (11) is surjective.

Let $\operatorname{Aut}(\mathcal{Q})$ be the group of holomorphic automorphisms of $\mathcal{Q}$. It is a complex Lie group with Lie algebra $H^{0}(\mathcal{Q}, T \mathcal{Q})$; as before, the Lie algebra operation on $H^{0}(\mathcal{Q}, T \mathcal{Q})$ is given by the Lie bracket of vector fields. The connected component of $\operatorname{Aut}(\mathcal{Q})$ containing the 
identity element will be denoted by $\operatorname{Aut}^{0}(\mathcal{Q})$. The following is an immediate consequence of Theorem 3.1 .

Corollary 3.2. The subgroup $\operatorname{PGL}(r, \mathbb{C}) \subset \operatorname{Aut}(\mathcal{Q})$ in $(10)$ coincides with $\operatorname{Aut}^{0}(\mathcal{Q})$.

\section{Torus aCtion on THE QUOT SCHEME}

Let $T \subset \operatorname{PGL}(r, \mathbb{C})$ be the maximal torus consisting of diagonal matrices. Restrict the action of $\operatorname{PGL}(r, \mathbb{C})$ on $\mathcal{Q}$ in $(\underline{10})$ to the subgroup $T$. Let

$$
\mathcal{Q}^{T} \subset \mathcal{Q}
$$

be the subset fixed pointwise by this action of $T$; it is a disjoint union of complex submanifolds of $\mathcal{Q}$. We will recall the description of the connected components of $\mathcal{Q}^{T}$ given in $[\mathrm{Bi}]$.

Consider a point of $\mathcal{Q}$ given by an exact sequence

$$
0 \longrightarrow \mathcal{F} \stackrel{\iota}{\longrightarrow} \mathcal{O}_{X}^{\oplus r} \longrightarrow Q \longrightarrow 0
$$

This exact sequence corresponds to a fixed point of the torus action on $\mathcal{Q}$ if and only if the homomorphism $\iota$ decomposes as

$$
\iota=\bigoplus_{j=1}^{r} \iota_{j}: \bigoplus_{j=1}^{r} \mathcal{L}_{j} \hookrightarrow \mathcal{O}_{X}^{\oplus r}
$$

where each $\iota_{j}$ is the inclusion of some ideal sheaf

$$
\iota_{j}: \mathcal{L}_{j} \hookrightarrow \mathcal{O}_{X}
$$

(see [Bi, p. 610]). We may write $\mathcal{L}_{j}=\mathcal{O}_{X}\left(-D_{j}\right)$, where $D_{j}$ is the divisor for $\iota_{j}$. We have

$$
\sum_{j=1}^{r} \operatorname{deg}\left(D_{j}\right)=d
$$

by the definition of $\mathcal{Q}$.

Denote by $\operatorname{Part}_{r}^{d}$ the set of partitions of $d$ of length $r$. So $\left(d_{1}, d_{2}, \cdots, d_{r}\right) \in \operatorname{Part}_{r}^{d}$ if and only if $d_{j}$ are nonnegative integers with

$$
\sum_{j=1}^{r} d_{j}=d
$$

By $\operatorname{Sym}^{0}(X)$ we will mean a point.

Proposition 4.1. The fixed point locus is a disjoint union

$$
\mathcal{Q}^{T}=\coprod_{\left(d_{1}, \cdots, d_{r}\right) \in \mathbf{P a r t}_{r}^{d}} \operatorname{Sym}^{d_{1}}(X) \times \cdots \times \operatorname{Sym}^{d_{r}}(X)
$$


Proof. If $r=1$, then $\operatorname{Quot}\left(\mathcal{O}_{X}, e\right)$ is the symmetric product $\operatorname{Sym}^{e}(X)$, as the map in (13) is an isomorphism. If for each $1 \leq i \leq m$,

$$
f_{i}: \mathcal{O}_{X} \longrightarrow Q_{i}
$$

is a torsion quotient of $\mathcal{O}_{X}$ of degree $e_{i}$, then

$$
\bigoplus_{i=1}^{m} f_{i}: \mathcal{O}_{X}^{\oplus m} \longrightarrow \bigoplus_{i=1}^{m} Q_{i}
$$

is a torsion quotient of $\mathcal{O}_{X}^{\oplus m}$ of degree $\sum_{i=1}^{m} e_{i}$. Therefore, for each partition

$$
\left(d_{1}, \cdots, d_{r}\right) \in \operatorname{Part}_{r}^{d}
$$

we have an inclusion map

$$
\operatorname{Sym}^{d_{1}}(X) \times \cdots \times \operatorname{Sym}^{d_{r}}(X) \hookrightarrow \mathcal{O}_{X}^{\oplus r} .
$$

It is clear that these subschemes together map onto the fixed point locus of the torus action. Further, the union is clearly disjoint.

The cohomology algebra of $\operatorname{Sym}^{e}(X)$ was computed by Macdonald [Ma, p. 325, (6.3)]. In particular, he showed that

$$
b_{1}\left(\operatorname{Sym}^{e}(X)\right):=\operatorname{dim} H^{1}\left(\operatorname{Sym}^{e}(X), \mathbb{Q}\right)=2 g .
$$

Consequently,

$$
b_{1}\left(\operatorname{Sym}^{d_{1}}(X) \times \cdots \times \operatorname{Sym}^{d_{r}}(X)\right)=2 g\left(\sum_{d_{i} \neq 0} 1\right) .
$$

Therefore, for elements $\left(d_{1}, \cdots, d_{r}\right) \in \operatorname{Part}_{r}^{d}$, the first Betti number $b_{1}\left(\operatorname{Sym}^{d_{1}}(X) \times \cdots \times\right.$ $\left.\operatorname{Sym}^{d_{r}}(X)\right)$ attains the minimum value if and only if some $d_{i}$ is $d$ and the rest are zero.

Hence Proposition 4.1 has the following corollary:

Corollary 4.2. Consider the first Betti number of the connected components of $\mathcal{Q}^{T}$. If the first Betti number of a connected component attains the minimum value, then this component is isomorphic to $\operatorname{Sym}^{d}(X)$.

Since any maximal torus of $\operatorname{PGL}(r, \mathbb{C})$ is conjugate to $T$, Proposition 4.1 and Corollary 4.2 remain valid if $T$ is replaced by any other maximal torus of $\operatorname{PGL}(r, \mathbb{C})$.

\section{The Torelli Theorem}

As before, $\mathcal{Q}:=\operatorname{Quot}_{X}\left(\mathcal{O}_{X}^{\oplus r}, d\right)$ is the Quot scheme with $d \geq 1$. If $g=2=d$, then we assume that $r>1$.

Let $X^{\prime}$ be a compact connected Riemann surface of genus $g^{\prime}$, with $g^{\prime} \geq 2$. Fix positive integers $r^{\prime}$ and $d^{\prime}$. If $g^{\prime}=2=d^{\prime}$, then we assume that $r^{\prime}>1$. Let

$$
\mathcal{Q}^{\prime}:=\operatorname{Quot}_{X^{\prime}}\left(\mathcal{O}_{X^{\prime}}^{\oplus r^{\prime}}, d^{\prime}\right)
$$

be the Quot scheme parametrizing the torsion quotients of $\mathcal{O}_{X^{\prime}}^{\oplus r^{\prime}}$ of degree $d^{\prime}$. 
Theorem 5.1. The complex manifolds $\mathcal{Q}$ and $\mathcal{Q}^{\prime}$ are biholomorphic if and only if the following conditions hold:

- $X$ is biholomorphic to $X^{\prime}$,

- $r=r^{\prime}$, and

- $d=d^{\prime}$.

Proof. Assume that $\mathcal{Q}$ is biholomorphic to and $\mathcal{Q}^{\prime}$. We will show that the three conditions in the theorem hold.

Fix a maximal torus

$$
T_{0} \subset \operatorname{Aut}^{0}(\mathcal{Q})
$$

where $\operatorname{Aut}^{0}(\mathcal{Q})$, as before, is the connected component the automorphism group of $\mathcal{Q}$ containing the identity element. Consider the action of $T_{0}$ on $\mathcal{Q}$. Let $\beta$ be the minimum value of the first Betti number of the connected components of the fixed point locus $\mathcal{Q}^{T_{0}}$. take a connected component $M \subset \mathcal{Q}^{T_{0}}$ such that

$$
b_{1}(M)=\beta .
$$

From Corollary 4.2 we know that $M=\operatorname{Sym}^{d}(X)$.

First assume that at least one of the following two conditions holds:

(1) $\operatorname{dim} M \neq 2$

(2) $b_{1}(M) \neq 4$.

These conditions imply that $g>2$ if $d=2$. Fakhruddin proved that for any compact connected Riemann surface $Y$, and for any positive integer $d$ such that $d \neq 2$ if $\operatorname{genus}(Y)=2$, the isomorphism class of $\operatorname{Sym}^{d}(Y)$ uniquely determines the isomorphism class of $Y\left[\mathrm{Fa}\right.$, Theorem 1]. From this we conclude that $X$ is isomorphic to $X^{\prime}$.

Considering the dimension of $T_{0}$ we conclude that $r=r^{\prime}$. Considering the dimension of $\mathcal{Q}$ we conclude that $d=d^{\prime}$.

Now consider the remaining case where

$$
\operatorname{dim} M=2=\frac{b_{1}(M)}{2} .
$$

Note that these imply that $g=2=d$. Hence $r \geq 2$ by the assumption.

Let $\widetilde{\beta}$ is the maximum value of the first Betti number of the connected components of the fixed point locus $\mathcal{Q}^{T_{0}}$. Let $\widetilde{M} \subset \mathcal{Q}^{T_{0}}$ be a connected component with

$$
b_{1}(\widetilde{M})=\widetilde{\beta} .
$$

From Proposition 4.1 and (17) we know that $\widetilde{M}=X \times X$.

Let $X$ and $Y$ be compact connected Riemann surfaces of genus two such that $X \times X$ is isomorphic to $Y \times Y$. Fixing an isomorphism $F: X \times X \longrightarrow Y \times Y$, consider the two maps

$$
X \longrightarrow Y, x \longmapsto f_{i} \circ F\left(x, x_{0}\right)
$$


where $f_{i}$ is the projection of $Y \times Y$ to the $i$-th factor. One of them is a nonconstant map, hence it is an isomorphism. Therefore, the isomorphism class of $X$ is uniquely determined by the isomorphism class of $X \times X$. This completes the proof.

Acknowledgements. The first author wishes to thank McGill University and University of Western Ontario for their hospitality.

\section{REFERENCES}

[Be] A. Bertram, Construction of the Hilbert scheme. Deformation theory I, http://www.math.utah.edu/ bertram/courses/hilbert.

[BDW] A. Bertram, G. Daskalopoulos and R. Wentworth, Gromov invariants for holomorphic maps from Riemann surfaces to Grassmannians, Jour. Amer. Math. Soc. 9 (1996), 529-571.

[Bi] E. Bifet, Sur les points fixes schéma Quot $_{\mathcal{O}_{X} / X, k}$ sous l'action du tore $\mathbf{G}_{m, k}^{r}$, Com. Ren. Acad. Sci. Paris 309 (1989), 609-612.

[Fa] N. Fakhruddin, Torelli's theorem for high degree symmetric products of curves, arxiv:0208180v1.

[GH] P. B. Griffiths and J. H. Harris, Principles of algebraic geometry, Pure and Applied Mathematics, Wiley-Interscience, New York, 1978.

[Ma] I. G. Macdonald, Symmetric products of an algebraic curve, Topology 1 (1962), 319-343.

School of Mathematics, Tata Institute of Fundamental Research, Homi Bhabha Road, BOMBAY 400005, INDIA

E-mail address: indranil@math.tifr.res.in

Department of Mathematics, Middlesex College, University of Western Ontario, LonDON, ON N6A 5B7, CANADA

E-mail address: adhill3@uwo.ca

Department of Mathematics, McGill University, Burnside Hall, 805 Sherbrooke St. W., Montreal, Que. H3A 0B9, Canada

E-mail address: jacques.hurtubise@mcgill.ca 